\title{
THE LORENTZ SPACE AS A DUAL SPACE
}

\author{
PRATIBHA G. GHATAGE
}

\begin{abstract}
If $(X, S, \mu)$ is a finite, completely nonatomic measure space and $\phi(t)=t^{1 / p}(p>1)$ then the Lorentz space $N_{\phi}$ is the dual space of the closed span of simple functions in $M_{\phi}\left(=N_{\phi}^{*}\right)$.
\end{abstract}

In this note we characterize the closed span of simple functions in $M_{\phi}$ and show that the Lorentz space $N_{\phi}$ is its dual. Though this result was inspired by the characterization of $H_{1}$ as the dual of $\mathrm{VMO}$, on account of the total lack of analyticity in the Lorentz space, the two methods have little in common.

Notation. Suppose $(X, S, \mu)$ is a completely nonatomic finite measure space, $\phi(t)=t^{1 / p}(p>1)$ and $N_{\phi}=\left\{f, f\right.$ measurable, $\left.\int_{0}^{\infty} \phi\left(\mu\left(F_{y}\right)\right) d y<\infty\right\}$, where $F_{y}=$ $\{x,|f(x)|>y\}$ for $y>0$. It is known that the dual space of $N_{\phi}$ is

$$
M_{\phi}=\left\{f, f \text { measurable, } \sup _{0<\mu(E)} \frac{1}{\phi(\mu(E))} \int_{E}|f(t)| d \mu<\infty\right\} .
$$

For more details, see [2].

Define

$$
M_{\phi}^{0}=\left\{f \in M_{\phi}, \lim _{\mu(E) \rightarrow 0} \frac{1}{\phi(\mu(E))} \int_{E}|f(t)| d \mu=0\right\} .
$$

LEMMA 1. $M_{\phi}^{0}=$ closed span of simple functions in $M_{\phi}$.

Proof. If $0<\mu(E)$ and $f=\psi_{E}$ then

$$
\sup _{F} \frac{1}{\mu(F)^{1 / p}} \int_{P}|f| d \mu=\sup _{F} \frac{\mu(F \cap E)}{\mu(F)^{1 / p}} \leq \sup _{F} \mu(F \cap E)^{1 / q} \leq \mu(E)^{1 / q},
$$

where $1 / p+1 / q=1$. Taking $F=E$ we see that $\|f\|_{M_{\phi}}=\mu(E)^{1 / q}$. Also

$$
\frac{1}{\mu(F)^{1 / p}} \int_{F}|f| d \mu=\frac{\mu(F \cap E)}{\mu(F)^{1 / p}} \leq \mu(F)^{1 / q} \text {. }
$$

Hence

$$
\lim _{\mu(F) \rightarrow 0} \frac{1}{\phi(\mu(F))} \int_{F}|f| d \mu=0 .
$$

It is easy to see that $M_{\phi}^{0}$ is a closed linear subspace of $M_{\phi}$ and hence contains simple functions.

Conversely suppose that $f \in M_{\phi}^{0}$. In particular $f \in M_{\phi}$ and hence $f \in L_{1}[\mathbf{2}$, Theorems 5.6, 5.7]. Let $\epsilon>0$. It follows by a standard approximation argument and the fact that $\mu$ is completely nonatomic [3, Theorems $1.17,3.13]$ that there

Received by the editors November 4, 1982 and, in revised form, January 10, 1983 and June 7, 1983.

1980 Mathematics Subject Classification. Primary 46E30. 
exists a sequence $\left\{f_{n}\right\}$ of simple functions such that $\left|f_{n}(x)\right| \leq|f(x)|$ a.e. for all $n$ and $\left\|f_{n}-f\right\|_{1} \rightarrow 0$. Choose $\delta$ which satisfies the property that whenever $0<$ $\mu(F)<\delta$,

$$
\frac{1}{\mu(F)^{1 / p}} \int_{F}|f| d \mu<\frac{\epsilon}{3} .
$$

(This is possible since $f \in M_{\phi}^{0}$.) By Egoroff's theorem there exists a measurable set $F$ with $\mu(F)<\delta$ and $f_{n} \rightarrow f$ uniformly on $X-F$. Choose $n_{0}$ such that

$$
\left|f_{n_{0}}(x)-f(x)\right|<\frac{\epsilon}{3(\mu(X))^{1 / q}} \quad \forall x \in X-F .
$$

Now whenever $0<\mu(E)$ we have

$$
\begin{aligned}
\frac{1}{\mu(E)^{1 / p}} \int_{E} & \left|f_{n}-f\right| d \mu=\frac{1}{\mu(E)^{1 / p}} \int_{E \cap F}\left|f_{n}-f\right| d \mu+\frac{1}{\mu(E)^{1 / p}} \int_{E-F}\left|f_{n}-f\right| d \mu \\
& \leq \frac{1}{\mu(E)^{1 / p}}\left[\int_{E \cap F}\left|f_{n}\right| d \mu+\int_{E \cap F}|f| d \mu\right]+\frac{1}{\mu(E-F)^{1 / p}} \int_{E-F}\left|f_{n}-f\right| d \mu \\
& \leq \frac{1}{(E \cap F)^{1-p}}\left[2 \int_{E \cap F}|f| d \mu\right]+\frac{\epsilon \mu(E-F)^{1 / q}}{3(\mu(X))^{1 / q}} \\
& \leq 2 \epsilon / 3+\epsilon / 3=\epsilon .
\end{aligned}
$$

Thus $\left\|f_{n_{0}}-f\right\|_{M_{\phi}} \leq \epsilon$ and this completes the proof.

THEOREM 1. The dual space of $M_{\phi}^{0}$ is isometrically isometric to $N_{\phi}$.

Proof. We first prove that given a bounded linear positive functional $T$ on $M_{\phi}^{0}$, there exists a function $k \in N$ such that $T f=\int_{X} k f d \mu$ and $\|k\|_{N_{\phi}} \leq\|T\|$. If $E$ is a $\mu$-measurable subset of $X$, define $m(E)=T\left(\psi_{E}\right)$. Note that $m(E) \leq$ $\|T\| \mu(E)^{1 / q}$ since $\left\|\psi_{E}\right\|_{M_{\phi}}=\mu(E)^{1 / q}$. It is easy to see that $m$ is a measure which is absolutely continuous with respect to $\mu$. If $k=d m / d \mu$ then $k \geq 0, k \in L_{1}(\mu)$ and $T f=\int_{X} k f d \mu$ for all simple functions $f$. If $y \geq 0$, define $F_{y}=\{x, k(x) \geq y\}$. In particular, $F_{n}=\{x, k(x) \geq n\}$. Then $\left\{F_{n}\right\}$ is a decreasing sequence and if $E_{n}=$ $F_{n}-F_{n+1},\left\{E_{n}\right\}$ is a sequence of pairwise disjoint measurable sets. Let

$$
f_{n}=\frac{\phi\left(\mu\left(F_{0}\right)\right)-\phi\left(\mu\left(F_{1}\right)\right)}{\mu\left(E_{0}\right)} \psi_{E_{0}}+\frac{\phi\left(\mu\left(F_{1}\right)\right)-\phi\left(\mu\left(F_{2}\right)\right)}{\mu\left(E_{1}\right)} \psi_{E_{1}}+\cdots+\frac{\phi\left(\mu\left(F_{n}\right)\right)}{\mu\left(E_{n}\right)} \psi_{E_{n}} .
$$

It is obvious that $f_{n} \in M_{\phi}^{0}$ and $\left\|f_{n}\right\|_{M_{\phi}} \leq 1$ [2, Theorem 5.5]. Hence $\|T\| \geq$ $\int_{X} k f_{n} d \mu$, but $\int_{E_{n}} k d \mu \geq n \mu\left(E_{n}\right)$. Hence

$$
\begin{aligned}
\|T\| \geq[ & \left.\phi\left(\mu\left(F_{1}\right)\right)-\phi\left(\mu\left(F_{2}\right)\right)\right]+\left[\phi\left(\mu\left(F_{2}\right)\right)-\phi\left(\mu\left(F_{3}\right)\right)\right] 2 \\
& +\cdots+\left[\phi\left(\mu\left(F_{n-1}\right)\right)-\phi\left(u\left(F_{n}\right)\right)\right](n-1)+n \phi\left(\mu\left(F_{n}\right)\right)
\end{aligned}
$$

i.e., $\|T\| \geq \phi\left(\mu\left(F_{1}\right)\right)+\phi\left(\mu\left(F_{2}\right)\right)+\cdots+\phi\left(\mu\left(F_{n}\right)\right)$ for all $n$. Thus $\sum_{k=1}^{\infty} \phi\left(\mu\left(F_{k}\right)\right) \leq$ $\|T\|$ and by the integral test $\int_{1}^{\infty} \phi\left(\mu\left(F_{y}\right)\right) d y \leq\|T\|$, i.e. $k \in N_{\phi}$ and a standard approximation argument shows that $\|k\|_{N_{\phi}} \leq\|T\|$.

On the other hand, for $k \in N_{\phi}$ and $f \in M_{\phi}$, we have $\left|\int k f d \mu\right| \leq\|k\|_{N_{\phi}}\|f\|_{M_{\phi}}[\mathbf{2}$, Theorem 4.4]. In particular, $\|T\| \leq\|k\|_{N_{\phi}}$. Thus there is an isometric isomorphism between the positive cones of $\left(M_{\phi}^{0}\right)^{*}$ and $N_{\phi}$ and the proof is complete. 


\section{REFERENCES}

1. N. Dunford and J. T. Schwartz, Linear operators. I, General Theory, Pure and Appl. Math., Vol. 7, Interscience, New York, 1958.

2. M. S. Steigerwalt and A. J. White, Some function spaces related to $L_{p}$ spaces, Proc. London Math. Soc. 22 (1971), 137-163.

3. W. Rudin, Real and complex analysis, 2nd ed., McGraw-Hill, New York, 1974.

Department of Mathematics, Cleveland State University, Cleveland, Ohio 4411.5 